Transport, stability and plasma control studies in the TJ-II stellarator

This content has been downloaded from IOPscience. Please scroll down to see the full text.

2015 Nucl. Fusion 55104014

(http://iopscience.iop.org/0029-5515/55/10/104014)

View the table of contents for this issue, or go to the journal homepage for more

Download details:

IP Address: 201.206.114.2

This content was downloaded on 21/03/2017 at 19:54

Please note that terms and conditions apply.

You may also be interested in:

Progress regarding magnetic confinement experiments, plasma-materials interactions and plasma performance

Carlos Hidalgo

Overview of TJ-II experiments

C. Hidalgo, C. Alejaldre, A. Alonso et al.

A review of impurity transport characteristics in the LHD

Shigeru Sudo

Dynamics of flows and confinement in the TJ-II stellarator

J. Sánchez, D. Alegre, A. Alonso et al.

Magnetic well scan and confinement in the TJ-II stellarator

Adriana M. de Aguilera, Francisco Castejón, Enrique Ascasíbar et al.

Experimental studies of the physical mechanism determining the radial electric field and its radial structure in a toroidal plasma

Katsumi Ida

Incompressibility of impurity flows in low density TJ-II plasmas and comparison with neoclassical theory

J. Arévalo, J.A. Alonso, K.J. McCarthy et al.

Compressible impurity flow in the TJ-Il stellarator

J. Arévalo, J.A. Alonso, K.J. McCarthy et al. 


\title{
Transport, stability and plasma control studies in the TJ-Il stellarator
}

\author{
J. Sánchez ${ }^{1}$, D. Alegre ${ }^{1}$, A. Alonso ${ }^{1}$, J. Alonso ${ }^{1}$, P. Alvarez ${ }^{1}$, J. Arévalo ${ }^{1}$, \\ E. Ascasíbar ${ }^{1}$, A. Baciero ${ }^{1}$, D. Baiao ${ }^{2}$, J.M. Barcala ${ }^{1}$, E. Blanco ${ }^{1}$, M. Borchardt ${ }^{3}$, \\ J. Botija ${ }^{1}$, S. Cabrera ${ }^{1}$, E. de la Cal ${ }^{1}$, I. Calvo ${ }^{1}$, A. Cappa ${ }^{1}$, R. Carrasco ${ }^{1}$, \\ F. Castejón ${ }^{1,4}$, R. Castro ${ }^{1}$, A. De Castro ${ }^{1}$, G. Catalán ${ }^{1}$, A.A. Chmyga ${ }^{5}$, \\ M. Chamorro ${ }^{1}$, A. Dinklage ${ }^{3}$, L. Eliseev ${ }^{6}$, T. Estrada ${ }^{1}$, F. Fernández-Marina ${ }^{1}$, \\ J.M. Fontdecaba ${ }^{1}$, L. García ${ }^{7}$, R. García-Gómez ${ }^{1}$, J.M. García-Regaña ${ }^{8,9}$, \\ J. Guasp ${ }^{1}$, R. Hatzky ${ }^{8}$, J. Hernández ${ }^{1}$, J. Herranz ${ }^{1}$, C. Hidalgo ${ }^{1}$, E. Hollmann ${ }^{10}$, \\ J.A. Jiménez ${ }^{1}$, A. Jiménez-Denche ${ }^{1}$, I. Kirpitchev ${ }^{1}$, R. Kleiber ${ }^{3}$, A.D. Komarov ${ }^{5}$, \\ A.S. Kozachok ${ }^{5}$, L. Krupnik ${ }^{5}$, F. Lapayese ${ }^{1}$, M. Liniers ${ }^{1}$, B. Liu ${ }^{1}$, D. López-Bruna ${ }^{1}$, \\ A. López-Fraguas ${ }^{1}$, J. López-Razola ${ }^{1}$, A. Martín de Aguilera ${ }^{1}$, F. Martín-Díaz, \\ F. Martín-Hernández ${ }^{1}$, A.B. Martín-Rojo ${ }^{1}$, J. Martínez-Fernández ${ }^{1}$, \\ K.J. McCarthy ${ }^{1}$, F. Medina ${ }^{1}$, M. Medrano ${ }^{1}$, L. Melón ${ }^{1}$, A.V. Melnikov ${ }^{6}$, P. Méndez $^{1}$, \\ B. van Milligen ${ }^{1}$, A. Molinero ${ }^{1}$, P. Monreal ${ }^{1}$, R. Moreno ${ }^{1}$, M. Navarro ${ }^{1}$, \\ I.S. Nedzelskiy ${ }^{2}$, M.A. Ochando ${ }^{1}$, J. Olivares ${ }^{1}$, E. Oyarzábal ${ }^{1}$, J.L. de Pablos ${ }^{1}$, \\ L. Pacios ${ }^{1}$, I. Pastor ${ }^{1}$, M.A. Pedrosa ${ }^{1}$, A. de la Peña ${ }^{1}$, A. Pereira ${ }^{1}$, A. Petrov ${ }^{5}$, \\ S. Petrov ${ }^{11}$, A.B. Portas ${ }^{1}$, G. Rattá ${ }^{1}$, E. Rincón ${ }^{1}$, L. Ríos ${ }^{1}$, C. Rodríguez ${ }^{1}$, B. Rojo ${ }^{1}$, \\ A. $\operatorname{Ros}^{1}$, M. Sánchez ${ }^{1}$, E. Sánchez ${ }^{1}$, E. Sánchez-Sarabia ${ }^{1}$, K. Sarksian ${ }^{12}$, \\ S. Satake ${ }^{13}$, J.A. Sebastián ${ }^{1}$, C. Silva ${ }^{1}$, E.R. Solano ${ }^{1}$, A. Soleto ${ }^{1}$, B. Sun ${ }^{1}$, \\ F.L. Tabarés ${ }^{1}$, D. Tafalla ${ }^{1}$, S. Tallents ${ }^{14}$, A. Tolkachev ${ }^{1}$, J. Vega ${ }^{1}$, G. Velasco ${ }^{1}$, \\ J.L. Velasco ${ }^{1,4}$, G. Wolfers ${ }^{1}$, M. Yokoyama ${ }^{13}$ and B. Zurro ${ }^{1}$ \\ ${ }^{1}$ Laboratorio Nacional de Fusión, CIEMAT, Madrid, Spain \\ ${ }^{2}$ Instituto de Plasmas e Fusao Nuclear, IST, Lisbon, Portugal \\ ${ }^{3}$ Max-Planck-Institut für Plasmaphysik, Greifswald, Germany \\ ${ }^{4}$ Instituto de Biocomputación y Física de Sistemas Complejos, Zaragoza, Spain \\ ${ }^{5}$ Institute of Plasma Physics, NSC KIPT, 310108 Kharkov, Ukraine \\ ${ }^{6}$ Institute of Nuclear Fusion, RNC Kurchatov Institute, Moscow, Russia \\ ${ }^{7}$ Universidad Carlos III, Madrid, Spain \\ ${ }^{8}$ Max-Planck-Institut für Plasmaphysik, Garching, Germany \\ ${ }^{9}$ EUROfusion PMU Garching, Boltzmannstr 2, D-85748, Garching, Germany \\ ${ }^{10}$ University of California, San Diego, CA, USA \\ ${ }^{11}$ A.F. Ioffe Physical Technical Institute, St Petersburg, Russia \\ ${ }^{12}$ General Physics Institute, Russian Academy of Sciences, Moscow, Russia \\ ${ }^{13}$ National Institute for Fusion Science, Toki, Japan \\ ${ }^{14}$ Instituto Tecnológico de Costa Rica, Cartago, Costa Rica \\ E-mail: Joaquin.sanchez@ciemat.es
}

Received 20 November 2014, revised 7 April 2015

Accepted for publication 13 April 2015

Published 29 July 2015

\section{Abstract}

The main TJ-II results since 2012 are presented in this overview. Impurity confinement is studied showing an isotopic dependence of impurity confinement time, asymmetries in parallel impurity flows in TJ-II ion-root plasmas and impurity density asymmetries within a flux surface. In addition, first observations of electrostatic potential variations within the same magnetic flux surface are presented. Evidence of the impact of three-dimensional magnetic structures on plasma confinement and L- $\mathrm{H}$ transitions is also presented. The leading role of the plasma turbulence is emphasized by the observed temporal ordering of the limit cycle oscillations at the L-I-H transition. Comparative studies between tokamaks and stellarators have provided direct experimental evidence for the importance of multi-scale physics to unravel the impact of the isotope effect on transport. Novel solutions for plasma facing components based on the recently installed Li-liquid limiters (LLLs) have been developed on TJ-II, showing the self-screening effect of evaporating liquid lithium, protecting plasma-facing components against heat loads, and tritium inventory control. Regarding plasma stability, magnetic well scan experiments show that traditional stability criteria, on which 
the optimization of stellarator configurations is based, may miss some stabilization mechanisms. Further effects of ECRH on Alfvénic instabilities are investigated, showing that moderate off-axis ECH power deposition modifies the continuous nature of the Alfvén eigenmodes, and frequency chirping sets in. This result shows that ECH can be a tool for AE control that might be ITER and reactor-relevant.

Keywords: transport, stability, control

(Some figures may appear in colour only in the online journal)

\section{Introduction}

This work presents an overview of TJ-II results, taking advantage of the flexibility offered by stellarator devices, which makes them ideal laboratories to study the relation between magnetic topology, electric fields and transport. Recent improvements in plasma diagnostics at TJ-II have enhanced our understanding of the confinement properties of fusion plasmas. The TJ-II plasmas discussed in this paper were heated by electron cyclotron resonance heating $(\mathrm{ECRH})(2 \times 300 \mathrm{~kW}$ gyrotrons, at $53.2 \mathrm{GHz}, 2 \mathrm{nd}$ harmonic, $\mathrm{X}$-mode polarisation) and neutral beam injection (NBI). ECRH power was coupled to the plasma via two quasi-optical transmission lines, located at symmetric stellarator positions, equipped with internal steerable mirrors. Beams with up to $700 \mathrm{~kW}$ of port-through power at $33 \mathrm{kV}$ were injected into target plasmas created with one or both gyrotrons. The above referred improvements in plasma diagnostics include a spectrally resolved motional Stark effect diagnostic to quantify the magnitude and pitch of the magnetic field at 10 positions across the minor radius, the commissioning of a four-barrel cryogenic pellet injector for core fuelling studies, and the commissioning of a second Heavy Ion Beam Probe $\left(\mathrm{Cs}^{+}\right.$ions, $\left.E_{b}=125 \mathrm{keV}\right)$ to characterize zonal flow type structures in the plasma core region.

This paper is organised in several sections that deal with the main TJ-II research topics. Section 2 is devoted to particle, energy and impurity transport. The studies will show the importance of asymmetries in parallel impurity flows in TJ-II ion-root plasmas. Such asymmetries have been interpreted in terms of a compressible variation of the impurity flow field and hence of impurity density asymmetries within a flux surface. This result is reinforced by the first observations of electrostatic potential variations within the same magnetic flux, which is modelled on the basis of neoclassical Monte-Carlo calculations. The dependence of impurity confinement time on charge and mass has been also studied. Section 3 deals with the physics of momentum transport and isotope effect paying attention to the leading role of the plasma turbulence on $\mathrm{L}-\mathrm{H}$ transitions, based on the limit cycle oscillations (LCO). On top of that, comparative studies between tokamaks and stellarators have provided direct experimental evidence for the importance of multi-scale physics to unravel the impact of the isotope effect on transport. Section 4 includes the studies of power exhaust physics and the research on novel solutions for plasma facing components based on the use of liquid metals like Li and alloys. The TJ-II liquid metals programme addresses fundamental issues including the self-screening effect of evaporating liquid lithium, protecting plasma-facing components against heat loads, using the recently installed Li-liquid limiters (LLLs). Section 5 studies the operation of TJ-II in configurations with magnetic hill, showing stable plasmas in Mercier-unstable configurations. This result is relevant for the design of future stellarators, since relaxing the Mercier stability criterion will lead to the use of simpler magnetic field coils. Finally, section 6 deals with fast particle control. The experiments on the stabilization and control of Alfvén eigenmodes (AEs) using ECRH show results that this heating method can be a tool for AE control that might be ITER and reactor-relevant.

\section{Particle, energy and impurity transport}

Neoclassical predictions are the basis of core plasma scenario development for any stellarator. For this reason, TJ-II has participated in an inter-machine validation study of neoclassical transport modelling in medium to high density stellarator-heliotron plasmas [1]. The goal of this study was to compare neoclassical predictions for radial particle and energy transport with experimental estimates in selected discharges at high ion collisionality (i.e., NBI-heated). Reasonable agreement was found between experimental and neoclassical particle fluxes in the core region $(r / a<0.5)$, and a worse agreement was found between heat fluxes in that region. Similar results were obtained in electron cyclotron resonance (ECR)-heated TJ-II plasmas [2].

A relevant feature of these studies is that all neoclassical simulations were made with the DKES code [3], relying on a local ansatz for neoclassical transport and using the monoenergetic approach. We have used FORTEC-3D, a non-local and non-monoenergetic Monte Carlo neoclassical code [4], in order to check whether removing these hypotheses alters the predictions and brings them closer to (or further from) the experimental measurements [5]. The radial electric field is measured by means of Doppler reflectometry and charge exchange recombination spectroscopy (CXRS) diagnostic (C6+) and the experimental fluxes are estimated by means of a transport balance calculation using ASTRA code [6] to analyse those quasi-stationary discharges. What we observe in the TJ-II case (figure 1) is that non-local effects do not improve agreement between theory and experiment significantly, probably because of the low ion temperature. In the core region, where the radial electric field is small, the magnetic drift is comparable to the $E \times B$ drift and tends to make the radial electric field less negative and to reduce the ion energy flux. At large radii, the radial component of the magnetic drift enhances the fluxes and makes the radial electric field more negative. The particle flux does not change since it is set by the electrons in the ion root, and it does not depend strongly on the radial electric field. With respect to the comparison with the experiment, we observe good agreement for the particle flux in the core region $(r / a<0.5)$, and bad agreement for the energy flux. 

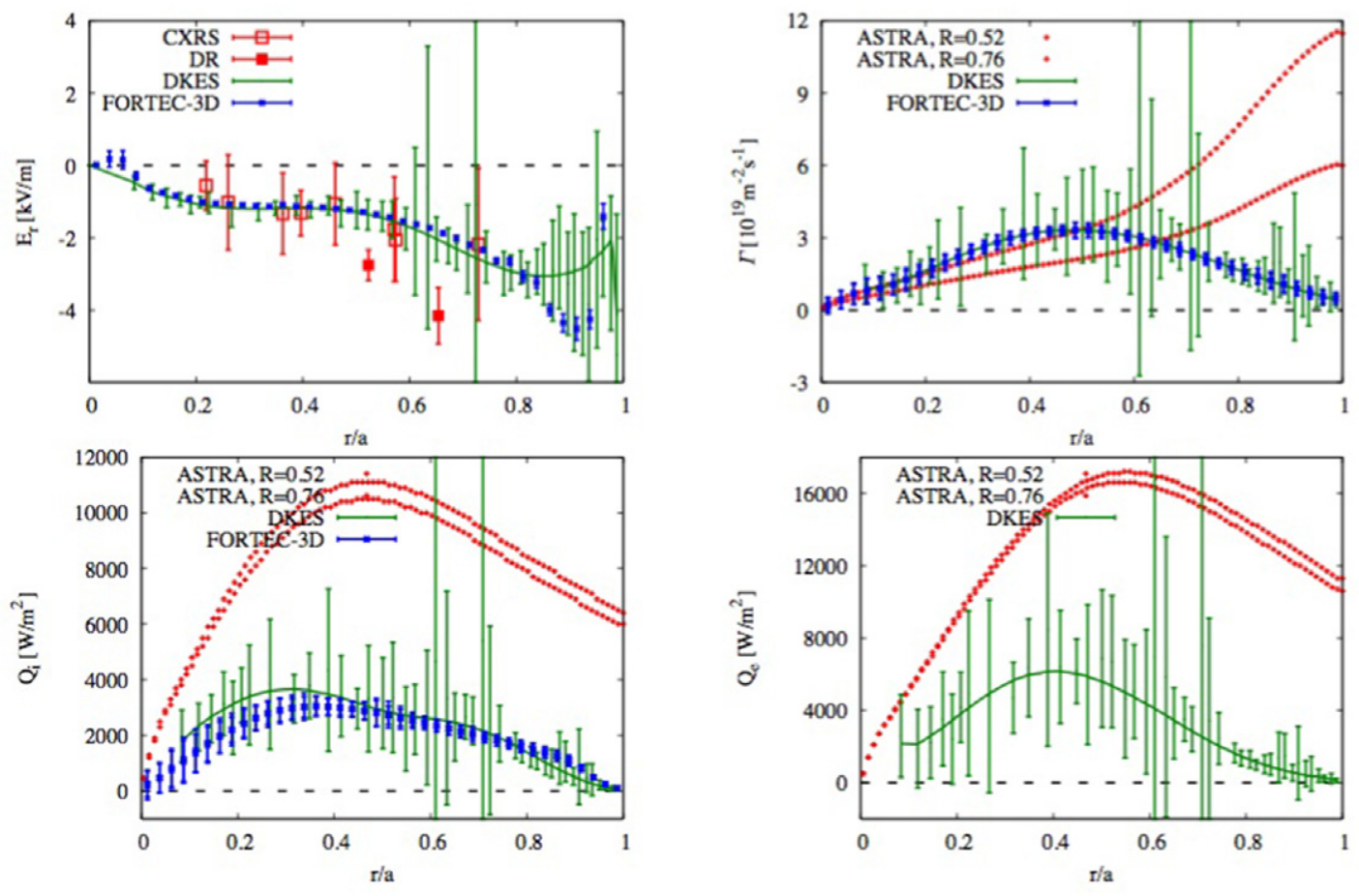

Figure 1. Result of the analysis of discharge \#28263: radial electric field (top left), particle flux (top right), ion energy flux (bottom left) and electron energy flux (bottom right). Two different values of the recycling factor $R$ were used in the ASTRA calculation in order to check the importance of the uncertainties in the plasma-wall interaction on the final results [5].

Neoclassical calculations predict also core impurity accumulation, which can hinder the achievement of long pulse high density plasmas in stellarators severely. The reduction of impurity influx from plasma facing components through the control of edge/scrape-off layer (SOL) plasma parameters and magnetic topology needs to be complemented with a more complete understanding of impurity transport processes in the confined region. Medium and high- $Z$ impurities are prone to develop significant density variations along a magnetic surface [7]. This can be due to their strong friction with ions, the angular variation of electrostatic potential, or their inertia. The variation in density and the presence of even a small poloidal electric field can modify flux surface averaged impurity fluxes with respect to standard neoclassical fluxes [8].

Impurity density asymmetries have been the subject of recent experimental and modelling efforts in the TJ-II stellarator [9]. Flow measurements obtained with CXRS diagnostic $\left(\mathrm{C}^{6+}\right)$ showed an incompressible parallel flow pattern in ECRH [10] and low-density NBI plasmas (figure 2 top). At higher densities, systematic variations of flow were observed within a magnetic surface (figure 2), which are interpreted as being due to particle number conservation in the presence of impurity density variations along the surface. Modelling based on the above-mentioned mechanisms (see [9] and references therein) resulted in density variations of 20 $30 \%$ for these higher density NBI plasmas, and return parallel flows of the correct size $\left(\sim 5 \mathrm{~km} \mathrm{~s}^{-1}\right)$. However, the calculated flow correction had the opposite sign of the observations. This disagreement has persisted, despite the inclusion of further ingredients in the model for parallel impurity force balance. Similar models for CXRS data in tokamaks have been unevenly successful $[11,12]$. This line of work is being continued at

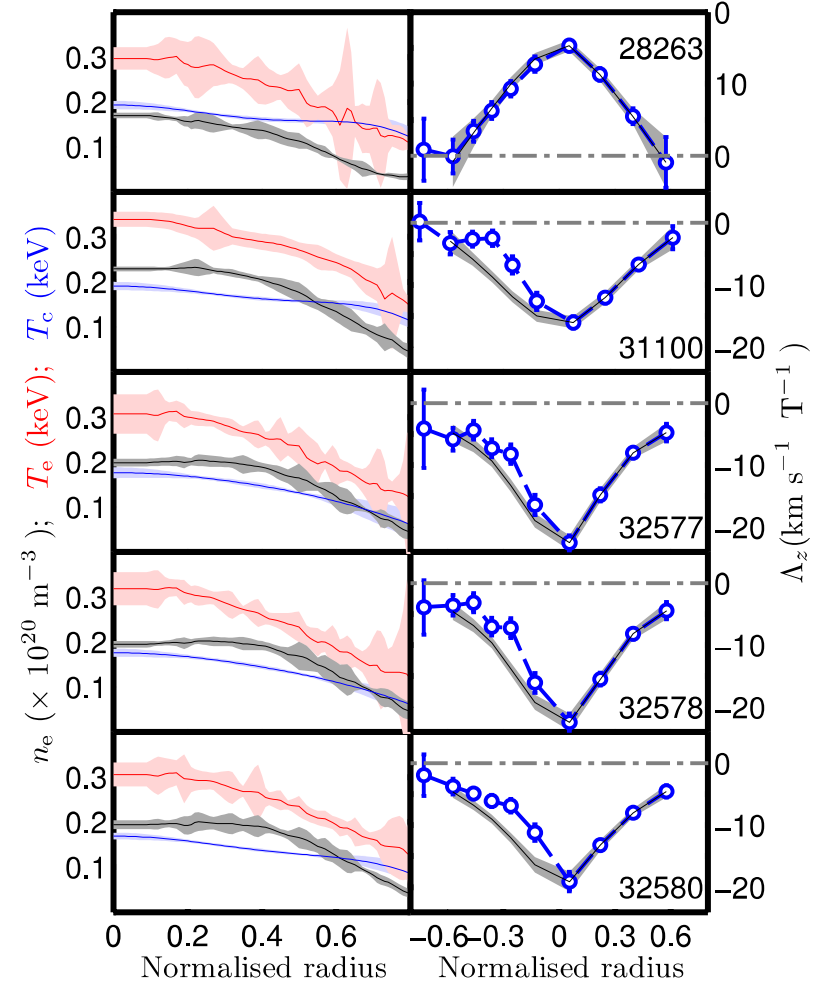

Figure 2. Left column: electron temperature ( $T_{\mathrm{e}}$ in red), density $\left(n_{\mathrm{e}}\right.$ in grey) and Carbon $6+$ temperature (CXRS, $T_{\mathrm{C}}$ in blue) profiles for several TJ-II discharges. Right column: net parallel flow of $\mathrm{C}^{6+}$ (after subtraction of Pfirsch-Schlüter flow) measured with CXRS (blue). An incompressible parallel flow should result in a symmetric profile (grey). Reproduced with permission from [9], copyright 2014 IAEA. 


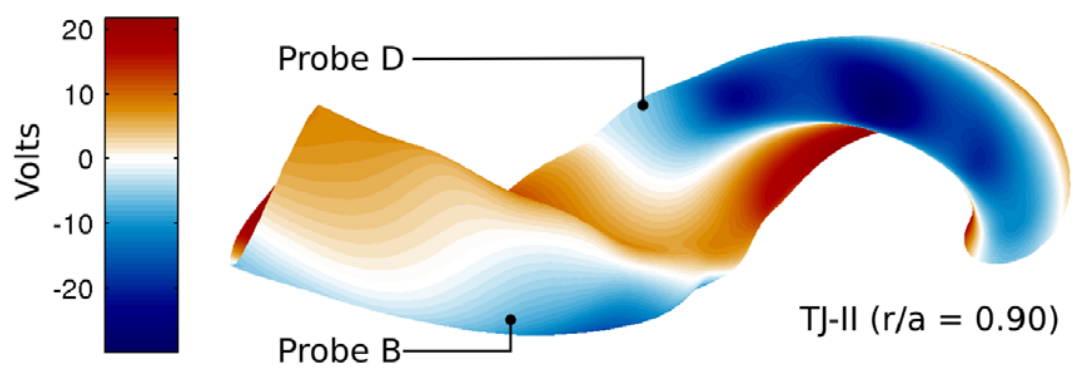

Figure 3. EUTERPE calculation of electrostatic potential variations over the flux surface $r / a=0.90$ for a typical ECRH, low density plasma. The position of the two Langmuir probe systems is indicated with lines.

TJ-II, checking measurements and simulations against other diagnostics and codes.

In parallel, duplicated Langmuir probe systems have been used to study the angular variation of the floating potential. The relative radial position of the probes is determined from the simultaneous detection of zonal flow-type structures [13,14]. Differences of several tens of volts are consistently observed in ECR-heated plasmas. Calculations of potential variations based on first order neoclassical quasi-neutrality arguments are being performed with the EUTERPE code (figure 3), as in [8].

The potential variation on flux surfaces $\Phi_{1}$ is commonly discussed in Neoclassical transport theory, as it enters the drift-kinetic equation for the first order correction $f_{\mathrm{i} 1}$ (in the usual Larmor radius expansion) to the Maxwellian distribution function $f_{\mathrm{i} 0}$. This variation is determined by first order quasineutrality, which results from balancing the adiabatic electron density variation on flux surfaces with the total ion density variation,

$$
\Phi_{1}=\frac{T_{\mathrm{e} 0}}{e} \frac{n_{\mathrm{i} 1}}{n_{\mathrm{e} 0}}
$$

$\Phi_{1}$ is small (order 1) in the normalised ion Larmor radius. Because of the angular dependency of radial drift, the first correction to the ion density is not constant on flux surfaces. For $\Phi_{1}=0$, the same correction for the electron density (which is order 1 in the electron Larmor radius) would be much smaller that the ion's and therefore unable to maintain plasma quasineutrality. The variation of potential $\Phi_{1}$ causes an adiabatic electron density variation that 'restores' quasineutrality. Finally, for stellarators this variation can grow considerably large due to the unfavourable scaling of $f_{\mathrm{i} 1}$ with the collisionality in non-axisymmetric systems for low collisionality regimes.

Simulations display peak-to-peak values of the order of magnitude of the experimental observations and also reproduce some qualitative dependencies of $\Phi_{1}$ with the radial electric field and electron temperature. However, for the specific location of the probes, the simulated differences are smaller than those observed experimentally [15] (see figure 3).

The impurity confinement time as a function of charge and mass of the impurity ions has been also studied [16]. Although an extensive data set does not follow the neoclassical $m^{1 / 2} / Z^{2}$ tendency well, some trends can be seen in figure 4 , which shows different behaviour for three impurities derived from soft $\mathrm{x}$-ray analysis and tomographic reconstructions (see figure 4(a)). In addition, impurity confinement exhibits a moderate isotope effect, decreasing as the $\mathrm{D}$ concentration increases, as can be seen in figure $4(b)$.

\section{Momentum transport and isotope physics}

Experiments and model development to improve the understanding of momentum transport and the physics of the confinement transition and intrinsic rotation are underway. TJ-II provides a clear evidence that three-dimensional magnetic structures have a significant impact on plasma confinement and $\mathrm{L}-\mathrm{H}$ transitions, and offers further insight into the radial propagation and dynamics of limit cycle oscillations at the L-I-H transition. Novel causality detection techniques were developed [17] and used in this study.

As an important topic regarding momentum transport and turbulence, non-linear coupling between turbulence and flows at the $\mathrm{L}-\mathrm{H}$ and $\mathrm{L}-\mathrm{I}-\mathrm{H}$ transitions was studied calculating the bicoherence of the turbulence. The bicoherence was found to peak at a specific radial position (slightly inward from the radial electric field shear layer in $\mathrm{H}$ mode). It was significantly enhanced in a time window of several milliseconds around the time of the $\mathrm{L}-\mathrm{H}$ transition and stayed high during the I-phase. The bicoherence is associated with a specific wave number range (intermediate, $\approx 8-11 \mathrm{~cm}^{-1}$ ) and due to the interaction between high frequencies and a rather low frequency, as indeed expected for a zonal flow [18].

During the I-phase, the temporal dynamics of the interaction between turbulence and flows displays LCO corresponding to a characteristic predator-prey relation between flows and turbulence. Recently, some controversial results were reported: two types of LCO were found in HL2A, with opposite temporal ordering [19]. The first type corresponds to the standard predator-prey model [20], where an increase of turbulence leads to the generation of a zonal flow, in turn suppressing the former. With the second type, the $E \times B$ flow grows first, causing the reduction of the fluctuations. The latter points to the pressure gradient as the potential drive for the oscillations, eventually inducing the transition to the H-mode [21].

At TJ-II, the turbulence flow front is found to propagate radially outwards at the onset of the LCO, but in some particular cases, after a short time interval without oscillations, a reversal in the front propagation velocity is seen [22]. Associated to this velocity reversal, a change in the temporal ordering of the LCO is observed when the turbulence level is represented versus $E \times B$ flow. However, the change in temporal ordering is not related to an intrinsic change in the nature of the LCO. By measuring simultaneously at three neighbouring radial positions with Doppler reflectometry [23], it has been possible to study the detailed evolution not only of the $E \times B$ flow but 

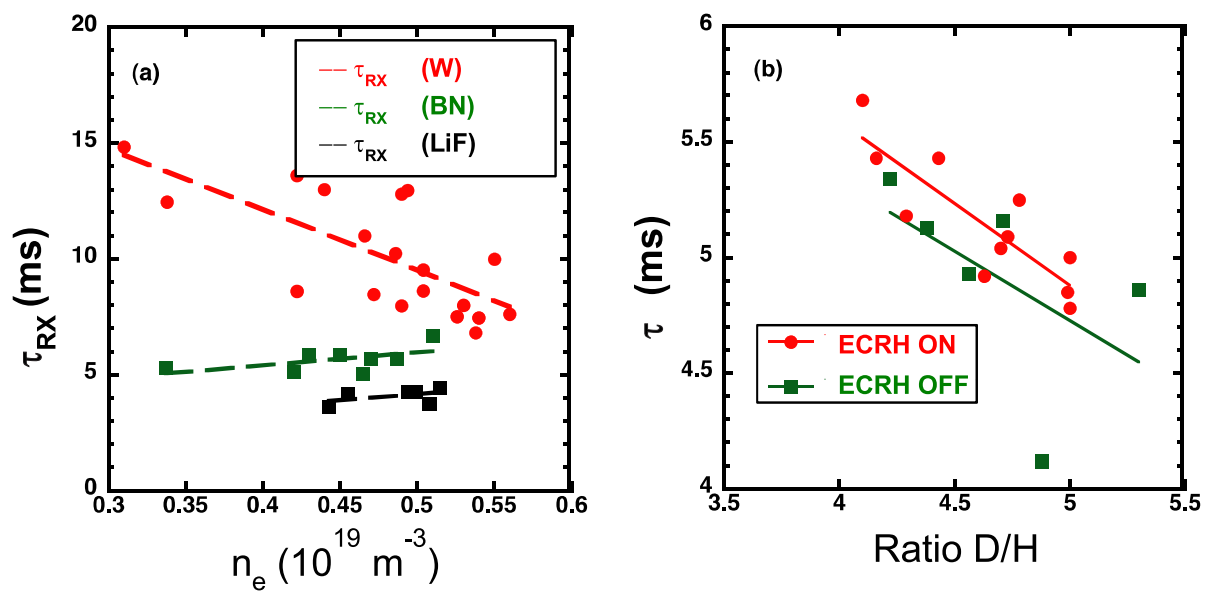

Figure 4. (a) Influence of the mass/charge ratio of the injected impurity on the relaxation decay time, for a central collimated x-ray detector. The decay time of soft $x$ ray is plotted versus average line density for tungsten $(\mathrm{W})$, boron nitride (BN) and lithium fluoride (LiF). An increase of the confinement time with mass and expected charge of the central ions is clearly seen when this data plotted as a function of the interferometer line-averaged density. (b) Dependence of the central impurity confinement time as function of the D/H ratio for $n_{\mathrm{e}}=(0.5 \pm 0.1) \times 10^{19} \mathrm{~m}^{-3}$ in NBI discharges with ECRH on and off [16].

also of the $E \times B$ flow shear and its relation with the turbulence level. As the turbulence-flow front propagates outwards, the increase in the turbulence level produces an increase in the inner (negative) $E \times B$ flow shear and when the propagation velocity reverses produces an increase in the outer (positive) $E \times B$ flow shear. In both cases of outward and inward propagating turbulent fronts, the turbulence increase leads and is followed by an increase in $E \times B$ flow shear, which in turn regulates the turbulence. TJ-II findings indicate that radial propagation mechanisms play an important role on the LCO dynamics, in addition to turbulence driven zonal flows and pressure gradients [24]. These results show that the L-H transition problem requires considering models including, at least, time dependency and one space dimension, as with the transition model reported in [25].

Dedicated experiments have been carried out to investigate the physical mechanisms triggering the onset and radial propagation of the LCO. At TJ-II, the LCOs are preferentially observed close to the transition threshold, in specific magnetic configurations having a low order rational close to the plasma edge. Preceding the onset of the oscillations, high frequency modes are often observed, accompanying a low frequency magnetohydrodynamic (MHD) mode. These modes are detected by the Doppler reflectometry system as oscillations of the centre of gravity $(\mathrm{CoG})$ of the spectra, a proxy for $E \times B$ flow, in the usual case of negligible contribution of turbulence phase velocity [25,26]. An example is shown in (figure 5). The results point to the interplay between the MHD mode and the $E \times B$ flow as a precursor for LCO onset at the L-I-H transition.

The possible relation between isotope effect and momentum transport and turbulence is also under investigation. Recent studies in the TEXTOR tokamak [27] and the TJ-II stellarator [28] report the properties of local turbulence and long-range-correlations (LRCs) in Hydrogen and Deuterium plasmas, concluding that there is a systematic increase in the LRC amplitude during the transition from $\mathrm{H}$ to $\mathrm{D}$ dominated plasmas in the TEXTOR tokamak, but not in the TJ-II stellarator. TJ-II investigations were done at the electron-ion root transition where there is a coupling between neoclassical viscosity and turbulent mechanisms [29]. Given the different scales acting on neoclassical and turbulent transport, this coupling provides the first direct experimental evidence of the importance of multi-scale physics for unravelling the impact of the isotope effect on transport in fusion plasmas. Gyrokinetic and semianalytical calculations are underway to explore the possible variation, if any, of zonal flow damping in TJ-II plasmas as a function of the different isotopes.

\section{Power exhaust physics}

With a view to steady state operation of burning plasmas, the selection of plasma facing materials for future Fusion devices remains challenging. Solid materials are known to be prone to disintegration, dust formation and neutron-induced permanent damage, among other deleterious effects [30]. Therefore, liquid metals, and lithium in particular, have been proposed as alternative to more conservative concepts [31]. One of the main concerns, however, is the survival of these elements to disruptions and type I edge-localized mode (ELMs), in particular considering strong MHD forces and massive evaporation. It has been shown that self-screening, driven by evaporation, could effectively protect liquid lithium elements against the huge heat loads occurring at the plasmasolid interface [32].

Based on previous findings of anomalous high secondary electron emission (SEE) of lithium surfaces exposed to a plasma [33], experiments were performed to bias the LLL [34], a $\mathrm{C}$ limiter and a solid lithium electrode, in order to test heat handling characteristics and the impact of injected currents on plasma performance while inserting the biased elements rather deeply into the plasma edge, which is not possible with other materials. Spontaneous currents develop at the plasma periphery when a $\mathrm{C}$ and a $\mathrm{Li}$ limiter are inserted at the same radial location, as a consequence of the very different SEE coefficients of both materials. Furthermore, the deduced electrical resistivity at the periphery is much higher than the Spitzer value, suggesting a direct relation 


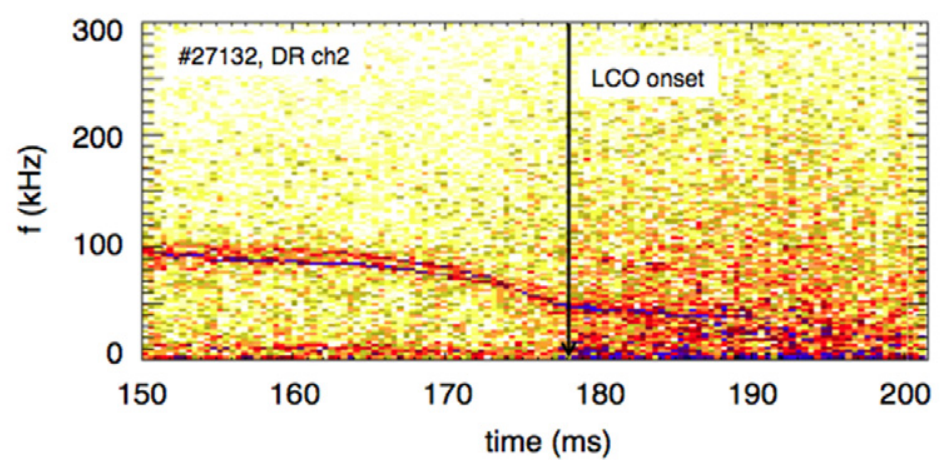

Figure 5. Spectrogram of the CoG of the Doppler reflectometer (a proxy for the $E \times B$ flow); the onset of the LCO is indicated with a vertical line.
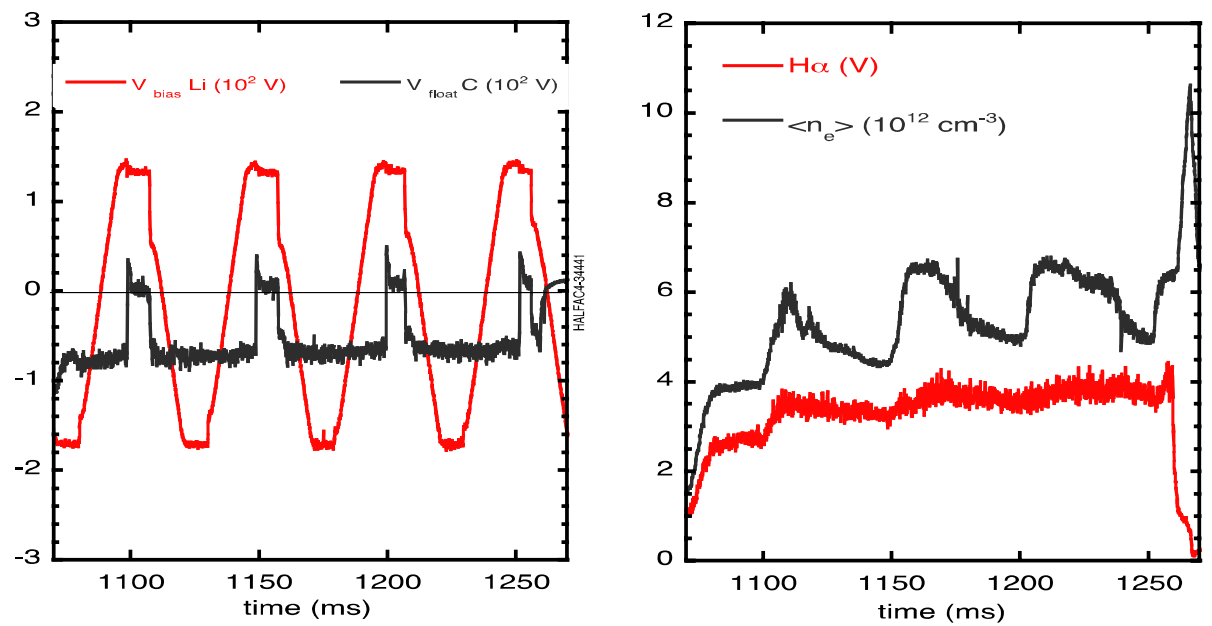

Figure 6. Effect of LLL bias on plasma parameters.

with the density of atomic species at the edge. In addition, inter-limiter biasing and limiter to vacuum vessel biasing experiments were performed, allowing the measurement of the electrical resistance at the edge. The results [35] show that LLL biasing is far more efficient to improve plasma confinement than $\mathrm{C}$, while no deleterious effect due to high power loads was seen. In contrast with other reports regarding the impact of limiter biasing on plasma confinement [36,37], positive biasing of lithium elements led to the enhancement of particle and energy confinement. Figure 6 (left) displays the response of the floating potential on the $\mathrm{C}$ limiter when the LLL is biased at $\pm 150 \mathrm{~V}$. As seen, the sheath potential on the floating limiter vanishes at maximum positive bias of the active limiter. When the situation was reversed, no changes on the floating potential of the LLL were detected. This asymmetric behaviour of the plasma under biasing of limiters of different materials is tentatively ascribed to the very high SEE of lithium surfaces exposed to plasma [33]. According to simple probe theory, a full suppression of the plasma sheath may take place for SEE values of the exposed surface near one [35]. However more experiments are still required to fully corroborate this hypothesis. The modulation of the electron density together with the $\mathrm{H} \alpha$ signal during the biasing of the LLL is shown in figure 6 (right). An increase by a $25 \%$ of the ratio ne/ $\mathrm{H} \alpha$, corresponding to particle confinement improvement, was deduced. No such improvement was seen in the reverse, $\mathrm{C}$ limiter biasing scenario (see [35] for details).
Power loads to the limiters were evaluated from the resulting surface temperature increase, leading to enhanced emission of $\mathrm{Li}$ atoms by evaporation, after in situ calibration of the lithium emission at selected temperatures of the LLL at early times of the discharge. Significantly lower power loads than those deduced from the He beam diagnostic were deduced at several insertion positions. This mismatch was ascribed to the presence of a power shielding mechanism by the $\mathrm{Li}$ vapour previously reported in similar experiments in other devices [32]. Outgassing of the LLL after exposure to the plasma indicates the release of a significant fraction of trapped $\mathrm{H}$ at temperatures below $400^{\circ} \mathrm{C}$, while no apparent $H / D$ retention was detected at $T_{\lim }=400^{\circ} \mathrm{C}$.

\section{Plasma stability studies}

At TJ-II, stable plasmas were achieved in Mercier unstable magnetic configurations without magnetic well, contrasting with MHD stability theory. The explanation is not clear yet, but the working theory is that the plasma tends to self-organize in the unstable regions, avoiding local instabilities [38]. This result is relevant for the design of new stellarators, since the magnetic well is strongly related to the plasma shape, which is created by complex coils. Thus, relaxing the Mercier stability criterion allows one to build simpler coils, which is easier and cheaper. Moreover, plasma shaping, i.e., triangularity and 


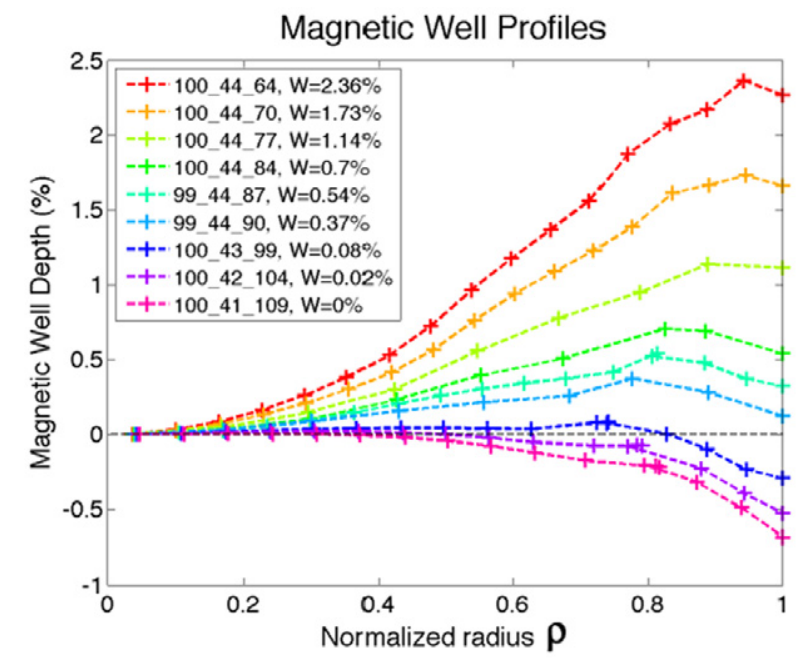

Figure 7. Values of the magnetic well for TJ-II configurations with rotational transform $8 / 5$ at $\rho=0.8$.

elongation, is known to affect tokamak stability (see [39] and references therein). These quantities are related to magnetic well, so these stellarator results can help to understand the role of shaping in tokamaks. The Mercier stability criterion is a measurement of plasma resilience against the interchange instability and can be written as follows [40]:

$$
D_{M}(\rho)=D_{W}(\rho)+D_{S}(\rho)+D_{I}(\rho)+D_{K}(\rho) \geqslant 0
$$

Where the first term on the rhs represents the stabilising contribution of the magnetic well. The remaining terms correspond to the shear, the plasma current and the geodesic curvature, respectively. TJ-II is an almost shearless stellarator, therefore the second and third terms are negligible and the Mercier stability properties are governed by the magnetic well and the curvature. TJ-II has the capability to vary its magnetic configuration, which allows one, for instance, to maintain the rotational transform profile almost constant while varying the magnetic well. Two families of configurations having such properties were chosen to perform the experiments presented here. One of them corresponds to configurations with $\iota / 2 \pi(\rho=0.8)=8 / 5$, while the other one corresponds to $\iota / 2 \pi(\rho=0.4)=3 / 2$. Figure 7 shows the values of the depth of the magnetic well for the configurations of the second family. The chosen heating method was NBI in order to obtain high density plasmas with significant $\beta$. In earlier work, a scan was performed across three Mercier stable configurations in electron cyclotron heating $(\mathrm{ECH})$ low density plasmas, showing an increase of the turbulent flux with decreasing well [41]. Here, we extend this work, operating TJ-II in unstable configurations. Stable confined plasmas are obtained even in Mercier unstable configurations with negative magnetic well, in both families. The preliminary results show that the confinement time, normalized to the plasma volume, is similar for all the configurations, once the radiated power is subtracted. It should be noted that plasma-wall interaction changes from one configuration to the next, which is taken into account by subtracting radiated power.

Plasma edge properties are studied with a Langmuir probe with nine pins, radially separated by $2 \mathrm{~mm}$ and measuring

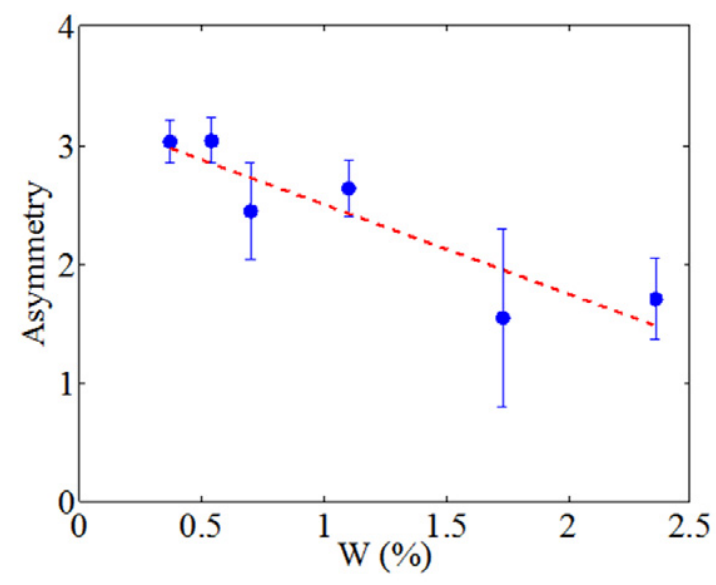

Figure 8. Flux-Gradient asymmetry versus radius versus magnetic well in the TJ-II stellarator.

floating potential. During the magnetic well scan, the shear layer tracks the position of the theoretical last closed flux surface (LCFS), showing that plasma edge confinement is not severely modified when magnetic well is reduced even when the configuration presents magnetic hill. Plasma turbulence changes as the scan is performed, as deduced from both probe measurements and fast cameras, such that fluctuations increase as the magnetic well is decreased. This effect is observed both in low and high density plasmas. Thomson scattering measurements indicate a flattening of pressure profiles in positions close to the rational surfaces, in some discharges without magnetic well or with magnetic hill, showing that the pressure profile reacts to mode destabilization, as also shown experimentally at the large helical device (LHD) [42].

Using edge Langmuir probes, the dynamical interaction between local particle fluxes $(\Gamma)$ and density gradients $(\nabla n)$ was studied during the magnetic well scan experiments. In this set of experiments, the magnetic configuration was scanned so as to vary the magnetic well at approximately constant effective probe position. The conditional expectation value of the flux, $E(\Gamma \mid \nabla n)$, was determined as in [43], showing a clear minimum in flux expectation at the most probable density gradient. A new quantifier was introduced to characterize the left-right asymmetry of this curve about this minimum: $A=$ $\Sigma E(\Gamma \mid \nabla n)[-\nabla n / \sigma(\nabla n)] / \Sigma E(\Gamma \mid \nabla n)$. Figure 8 shows how the asymmetry systematically decreases as the magnetic well is increased. Based on critical gradient transport models, these results are interpreted as follows. The edge region is assumed to be mostly supercritical due to the large drive, corresponding to large and positive asymmetry. However, at increased well, turbulence and instabilities are partly suppressed, bringing the plasma closer to a (self-organized) critical state. Thus, the observed flux-gradient dynamics provide support for critical gradient models of plasma transport.

\section{Fast particle physics}

The impact of ECR heating on NBI-driven Alfvén modes has been studied using different heating configurations. Several off-axis positions of the second beam $(\mathrm{ECH} 2)$ were tested while keeping the first beam (ECH1) fixed. As already reported in [44], launching at $\rho_{\mathrm{ECH} 2}=0.42$ produces strong 
33239

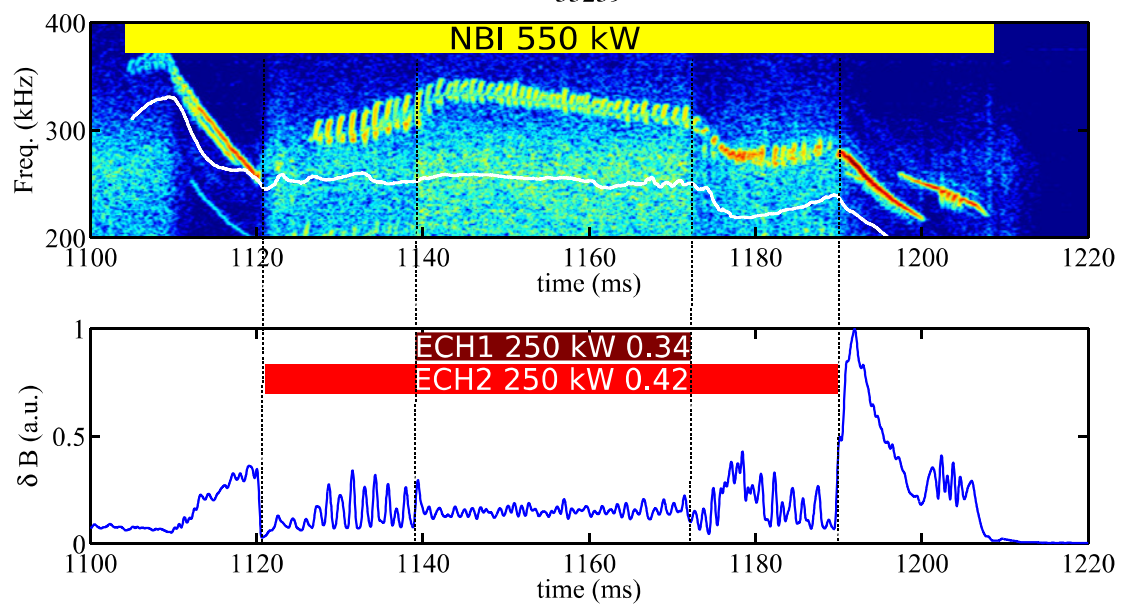

Figure 9. Steady NBI-driven mode (1110-1120 ms) and ECH2-induced chirping mode. The chirping mode amplitude is strongly reduced when ECH1 (1140-1172 ms) is added. Reproduced with permission from [44], copyright 2013 IAEA

mode chirping in a configuration with a medium value of the rotational transform in TJ-II. We take the configuration such that $\iota(0) / 2 \pi=1.55$, when the range of rotational transform achievable in TJ-II is $0.9<\iota(0) / 2 \pi<2.2$. Figure 9 illustrates the case in which $250 \mathrm{~kW}$ of $\mathrm{ECH} 2$, targeting the plasma at $\rho_{\mathrm{ECH} 2}=0.42$, is switched on again only $10 \mathrm{~ms}$ after the start of the NBI phase. As a consequence, the NBI driven steady mode disappears, then nothing is observed during a short period of time (5-7 ms) and then, a chirping mode with $\Delta f \approx 20 \mathrm{kHz}$ emerges. Note that ECRH induced pump-out helps controlling the density, which otherwise would increase gradually due to NBI particle fuelling. Adding further power using low frequency modulation of the first beam $(\mathrm{ECH} 1,250 \mathrm{~kW})$ at attenuates the amplitude of the chirping mode significantly, as clearly shown by the time evolution of the fluctuation amplitude $\delta B$, represented in figure 9 (bottom). The white line superimposed in figure 9 (top), which corresponds to $200 \times$ $\left(n_{\mathrm{L}}(t)\right)^{-1 / 2}$, where $n_{\mathrm{L}}(t)$ is the line average density, provides a rough indication of the frequency evolution expected from the theoretical Alfvén dispersion relation. Note that the line density is approximately constant during the transition from $\mathrm{ECH} 2$ to $\mathrm{ECH} 2+\mathrm{ECH} 1(t \approx 1140 \mathrm{~ms})$.

For $\rho_{\mathrm{ECH} 2} \leqslant 0.34$, frequency chirping is not observed and the steady NBI-driven mode is present. Again, adding the first beam at $\rho_{\mathrm{ECH} 1}=0.34$ causes a $50 \%$ reduction of the amplitude of the steady mode, relative to the case without ECRH. Together with this chirping mode, a reversal in the total plasma current $I_{\mathrm{p}}$ is observed. The plasma current changes its sign from $I_{\mathrm{p}}(t) \approx-0.6 \mathrm{kA}$ to $I_{\mathrm{p}}(t) \approx+0.7 \mathrm{kA}$ when the second beam is moved from 0.34 to 0.42 and therefore its absolute value barely changes. The density and temperature profiles show almost no variation in the gradient zone, where the contribution from the bootstrap current is expected to be larger and thus, the change of the plasma current cannot be explained by modifications in the plasma profiles.

Coincident with ECH injection, the neutral flux measured by a 16-channel compact neutral particle analyser (CNPA) is observed to be enhanced. This is consistent with an increase in the fast ion population during the $\mathrm{ECH}$ phase. Two alternative magnetic configurations with lower and higher $\iota$ than the above mentioned one were also investigated. Preliminary results indicate that the $\mathrm{ECH}$ off-axis position for maximum mode chirping depends on the configuration.

\section{Acknowledgments}

This work has been partially funded by the Spanish Ministry of Economy and Competitiveness under contract numbers ENE2013-48109-P, ENE2012-30832, ENE2012-38620-C0201, ENE2010-19109 and by the US Department of Energy under DE-FG02-07ER54917.

This work has been carried out within the framework of the EUROfusion Consortium and has received funding from the European Union's Horizon 2020 research and innovation programme under grant agreement number 633053 . The views and opinions expressed herein do not necessarily reflect those of the European Commission.

\section{References}

[1] Dinklage A. et al 2013 Inter-machine validation study of neoclassical transport modeling in medium- to high-density stellarator-heliotron, plasmas Nucl. Fusion $\mathbf{5 3} 063022$

[2] Tallents S. et al 2014 Transport analysis in an ECH power scan of TJ-II plasmas Plasma Phys. Control. Fusion $\mathbf{5 6} 075024$

[3] Hirshman S.P., Shaing K.C., van Rij W.I., Beasley C.O. and Crume E.C. 1986 Plasma transport coefficients for nonsymmetric toroidal confinement systems Phys. Fluids 292951

[4] Satake S., Okamoto M., Nakajima N., Sugama H. and Yokoyama M. 2006 Non-local simulation of the formation of neoclassical ambipolar electric field in non-axisymmetric configurations Plasma Fusion Res. 1002

[5] Velasco J.L. et al 2014 Validation of local and non-local neoclassical predictions for the radial transport of plasmas of low ion collisionallity 41st EPS Conf. on Plasma Physics (Berlin, Germany) http://ocs.ciemat.es/ EPS2014PAP/pdf/P2.077.pdf

[6] Pereverzev G.V. and Yushmanov P.N. 2002 ASTRA automated system for transport analysis Report IPP 5/98 (Germany, Garching: Max-Planck-Institut für Plasmaphysik)

[7] Helander P. 1998 Bifurcated neoclassical particle transport Phys. Plasmas 53999

[8] García-Regaña J.M. et al 2013 On neoclassical impurity transport in stellarator geometry Plasma Phys. Control. Fusion 55074008 
[9] Arévalo J. et al 2014 Compressible impurity flow in the TJ-II stellarator Nucl. Fusion $\mathbf{5 4 0 1 3 0 0 8}$

[10] Arévalo J. et al 2013 Incompressibility of impurity flows in low density TJ-II plasmas and comparison with neoclassical theory Nucl. Fusion $\mathbf{5 3} 023003$

[11] Reinke M.L. et al 2012 Poloidal variation of high-Z impurity density due to hydrogen minority ion cyclotron resonance heating on Alcator C-Mod Plasma Phys. Control. Fusion 54045004

[12] Viezzer E. et al 2013 Rotation and density asymmetries in the presence of large poloidal impurity flows in the edge pedestal Plasma Phys. Control. Fusion 55124037

[13] Pedrosa M.A. et al 2008 Evidence of long-distance correlation of fluctuations during edge transitions to improved-confinement regimes in the TJ-II stellarator Phys. Rev. Lett. 100215003

[14] Alonso A. et al 2012 Dynamic transport regulation by zonal flow-like structures in the TJ-II stellarator Nucl. Fusion 52063010

[15] Pedrosa M.A., Alonso J.A., García-Regaña J.M., Hidalgo C., Velasco J.L., Calvo I., Silva C. and Helander P. 2015 Electrostatic potential variations along flux surfaces in stellarators Nucl. Fusion 55052001

[16] Zurro B. et al 2014 Studying the impurity charge and main ion mass dependence of impurity confinement in ECR-heated TJ-II stellarator plasmas Plasma Phys. Control. Fusion 56124007

[17] van Milligen B. et al 2014 Causality detection and turbulence in fusion plasmas Nucl. Fusion 54023011

[18] van Milligen B. et al 2013 Spatiotemporal and wavenumber resolved bicoherence at the low to high confinement transition in the TJ-II stellarator Nucl. Fusion $\mathbf{5 3} 113034$

[19] Cheng J. et al 2013 Dynamics of low-intermediate-high-confinement transitions in toroidal plasmas Phys. Rev. Lett. 110265002

[20] Kim E.J. and Diamond P.H. 2003 Zonal flows and transient dynamics of the L-H transition Phys. Rev. Lett. 90185006

[21] Itoh S.I. and Itoh K. 1988 Model of L to H-Mode transition in tokamak Phys. Rev. Lett. 602276

[22] Estrada T. et al 2011 Spatiotemporal structure of the interaction between turbulence and flows at the $\mathrm{L}-\mathrm{H}$ transition in a toroidal plasma Phys. Rev. Lett. 107245004

[23] Happel T. et al 2009 Doppler reflectometer system in the stellarator TJ-II Rev. Sci. Instrum. 80073502

[24] Estrada T. et al 2015 Limit cycle oscillations at the L-I-H transition in TJ-II plasmas: triggering, temporal ordering and radial propagation Nucl. Fusion $\mathbf{5 5} 063005$

[25] Miki K. et al 2012 Spatio-temporal evolution of the L.H transition Phys. Plasmas 19092306

[26] Estrada T. et al 2012 A new approach to detect coherent modes using microwave reflectometry Nucl. Fusion $\mathbf{5 2} 082002$
[27] Xu Y. et al 2013 Isotope effect and multiscale physics in fusion plasmas Phys. Rev. Lett. 110265005

[28] Liu B. et al 2014 Isotope effect physics, turbulence and long-range correlation studies in the TJ-II stellarator 41 st EPS Conf. on Plasma Physics (Berlin, Germany)

[29] Velasco J.L. et al 2012 Vanishing neoclassical viscosity and physics of the shear layer in stellarators Phys. Rev. Lett. 109135003

[30] Linke J. 2006 High heat flux performance of plasma facing materials and components under service conditions in future fusion reactors Fusion Sci. Technol. 49455

[31] Mohamed A. and Abdou M.A. 2004 On the exploration of innovative concepts for fusion chamber technology Fusion Eng. Des. 72

[32] Apicella M.L. et al 2012 Lithization of the FTU tokamak with a critical amount of lithium injection Plasma Phys. Control. Fusion 54035001

[33] Oyarzabal E. et al 2014 Strongly enhanced secondary electron emission of Li surfaces exposed to low pressure plasmas $J$. Nucl. Mater. 45237

[34] Vertkov A.V. et al 2012 Status and prospect of the development of liquid lithium limiters for stellarotor TJ-II Fusion Eng. Des. 871755

[35] Tabares F.L. et al 2014 First liquid lithium limiter biasing experiments in the TJ-II stellarator J. Nucl. Mater. 463 1142-6

[36] Hidalgo C. et al 2004 Improved confinement regimes induced by limiter biasing in the TJ-II stellarator Plasma Phys. Control. Fusion 46287

[37] Weynants R.R. and van Oost G. 1993 Edge biasing in Tokamaks Plasma Phys. Control. Fusion 35 B177

[38] Ichiguchi K. and Carreras B.A. 2011 Multi-scale MHD analysis incorporating pressure transport equation for beta-increasing LHD plasma Nucl. Fusion $\mathbf{5 1} 053021$

[39] Doyle E.J. et al 2007 Progress in the ITER physics basis: chapter 2. Plasma confinement and transport Nucl. Fusion $47 \mathrm{~S} 18$

[40] Shafranov V.D. 1883 Magnetohydrodynamic theory of plasma equilibrium and stability in stellarators: a survey of results Phys. Fluids $\mathbf{2 6} 357$

[41] Castellano J. et al 2002 Magnetic well and instability thresholds in the TJ-II stellarator Phys. Plasmas 9713

[42] Sakakibara S. et al 2013 Response of MHD stability to resonant magnetic perturbation in the large helical device Nucl. Fusion 53043010

[43] Hidalgo C. et al 2012 Dynamical coupling between gradients and transport in fusion plasmas Phys. Rev. Lett. 108065001

[44] Nagaoka K. et al 2013 Mitigation of NBI-driven Alfvén eigenmodes by electron cyclotron heating in the TJ-II stellarator Nucl. Fusion $\mathbf{5 3} 072004$ 